\title{
Statins and Diabetes: Evaluating the Truth?
}

Arden R. Barry PharmD, Glen J. Pearson PharmD

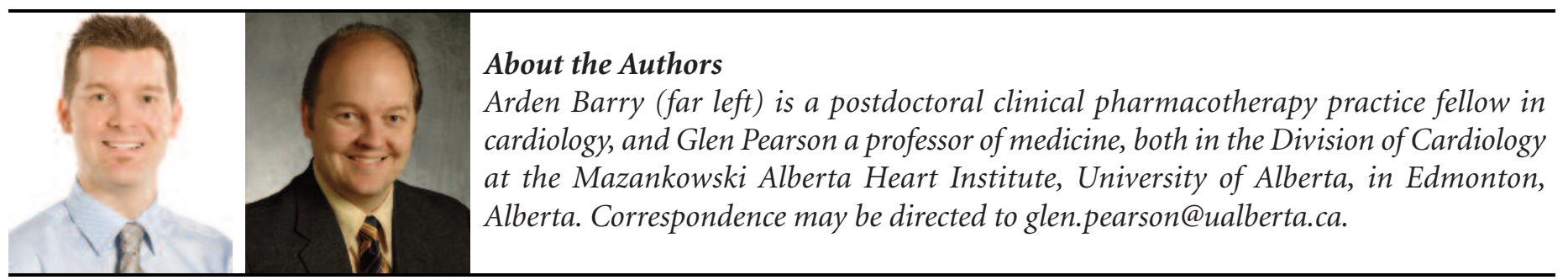

\section{Summary}

We are currently faced with yet another controversy surrounding an exceedingly common pharmacological therapy: are statins associated with an increased incidence of diabetes? In this article, the authors aim to explore the truth behind this recently contentious debate.

\section{Résumé}

Nous sommes de nouveau devant une autre question controversée touchant une thérapie pharmacologique extrêmement courante : les statines sont-elles associées à l'augmentation de l'incidence du diabète? Dans cet article, les auteurs explorent les éléments de vérité qui soustendent ce questionnement récent et controversé.

To take inspiration from the fictional character Colonel Nathan Jessup:

\begin{abstract}
We live in a world of cardiovascular disease. And that cardiovascular disease has to be treated with drugs like statins. Though to us the idea that statins cause diabetes is grotesque and incomprehensible, the truth is that statins save lives. Clinicians want statins to treat dyslipidemia; we need statins to treat dyslipidemia. We use words in medicine like "absolute risk reduction," "allcause mortality," and "risk versus benefit," and we use these words because evidence-based medicine is the backbone of our practice. Statins benefit patients with cardiovascular disease. So are we now justified in questioning that very benefit by claiming statins cause diabetes? Can we handle the truth?
\end{abstract}

Jack Nicholson's infamous monologue and line "You can't handle the truth!" from the motion picture A Few Good Men resonates deeply in today's popular culture. Tell someone, "I want the truth!" and most individuals know what you are referring to. Some may agree this line is also immensely pertinent in health care. The pursuit of the truth about drug therapy is just as relevant in medicine as it is in a military courtroom. We are currently faced with yet another controversy surrounding an exceedingly common pharmacological therapy, and our desire for the truth is no less compelling: are statins associated with an increased incidence of diabetes? In this article, we aim to explore the truth behind this recently contentious debate.

The association between statins and new-onset diabetes has been highly publicized in the medical literature, as well as in the mainstream news media. A recent Canadian Broadcasting Corporation (CBC) News article, published on January 10, 2012, contained the headline: "Statins Linked with Small Diabetes Risk." ${ }^{1}$ However, this is not a "new side-effect," as was stated in the article. The potential link between diabetes and statins, based on large randomized controlled trials, has been known for years. The reason this potential relationship was never determined to be conclusive is that the trials were not designed to detect differences in rates of diabetes, and therefore the play of chance could not be ruled out. However, a couple of recently published articles, as well as a safety communication from the Food and Drug Administration (FDA) in the United States, have escalated this debate.

Published in 2008, the Justification for the Use of Statins in Prevention: an Intervention Trial Evaluating Rosuvastatin (JUPITER) was the largest placebo-controlled trial to demonstrate an increased incidence of diabetes with statin therapy. ${ }^{2}$ The study enrolled 17,802 men and women with no previous history of cardiovascular $(\mathrm{CV})$ disease to rosuvastatin 
$20 \mathrm{mg}$ PO daily or placebo. The study was discontinued prematurely after 2 years as the rate of the primary outcome (CV death, myocardial infarction [MI], stroke, arterial revascularization, or hospitalization for unstable angina) was statistically significantly lower in the rosuvastatin group $(1.6 \%$ versus $2.8 \%$, hazard ratio [HR] $0.56,95 \%$ confidence interval [CI] $0.46-0.69, p<.001)$. Additionally, there was a significant reduction in death, from any cause, of $0.6 \%$ (2.2\% versus $2.8 \%$, $p=.02)$. However, there was also a significant increase in the rate of new-onset diabetes with rosuvastatin by $0.6 \%(3.0 \%$ versus $2.4 \%, p=.01)$. The incidence of diabetes was based on physician reporting and was not adjudicated by the events committee. Therefore, the authors speculated that reporting bias may have contributed to this difference. Furthermore, they stated that the protocol-driven monitoring of fasting blood glucose levels was not different between groups. Overall, if one were to compare the benefit versus risk of rosuvastatin in the JUPITER study, the number needed to treat (NNT) to prevent one adverse $\mathrm{CV}$ event was 167 per year and the number needed to harm (NNH) for incident diabetes was 334 per year. Consequently, for every 1,000 patients treated for 1 year with rosuvastatin, one would expect to prevent about six CV events and cause about three new cases of diabetes.

This controversy was fueled by a post hoc analysis of the Stroke Prevention by Aggressive Reduction in Cholesterol Levels (SPARCL) trial, which investigated atorvastatin $80 \mathrm{mg}$ daily versus placebo for 4.9 years in patients with a recent ischemic stroke or transient ischemic attack. ${ }^{3}$ The results demonstrated an absolute risk increase (ARI) in new-onset type 2 diabetes of $2.7 \%$ (HR 1.37, 95\% CI 1.08-1.75). However, two other trials that compared atorvastatin $80 \mathrm{mg}$ to other less potent statin therapy did not show a statistical difference in the rate of diabetes. Analysis of all three trials demonstrated that baseline fasting blood glucose, body mass index, hypertension, and fasting triglycerides were all independent risk factors for the development of type 2 diabetes. ${ }^{3}$ Thus, patients that developed diabetes while on statin therapy already had risk factors at baseline, and a higher number of risk factors corresponded to an increased risk.

In 2010, Sattar et al. published a meta-analysis of large randomized controlled trials investigating the link between statins and diabetes in an attempt to more definitively answer this question. ${ }^{4}$ This meta-analysis included trials that investigated statins versus placebo or no treatment and that reported clinically relevant CV outcomes. Included trials must have enrolled over 1,000 patients with follow-up data of more than 1 year. The search strategy yielded 13 studies, including the JUPITER study, that provided data for 91,140 primary and secondary prevention patients over a mean follow-up of 4 years.

The authors were able to obtain unpublished data on incident diabetes for seven of the included trials. Overall, the use of statins was associated with a statistically significantly higher rate of diabetes, with an odds ratio (OR) of 1.09 (95\% CI 1.02-1.17). The authors concluded that statin therapy over the course of 4 years would result in one new diagnosis of diabetes per 255 patients. Therefore, if 1,000 patients were treated with a statin for 4 years, about four patients would develop incident diabetes secondary to statin therapy (approximately one patient per year). The authors judged that the likelihood of this being a chance finding was low, as the results were associated with low statistical heterogeneity $\left(I^{2}=\right.$ $11.2 \%)$.

In 2011, Preiss et al. published a meta-analysis investigating the comparison between "intensive-dose" and "moderate-dose" statin therapy. ${ }^{5}$ As with the previous meta-analysis by Sattar et al., included were trials that enrolled over 1,000 patients with more than 1 year of follow-up. All patients included had a history of coronary artery disease (CAD) - as a recent acute coronary syndrome, previous MI, or stable CAD. The search strategy identified five trials. Intensive-dose statin therapy included three trials of atorvastatin $80 \mathrm{mg}$ daily and two of simvastatin $80 \mathrm{mg}$ daily. Moderate-dose comparators included atorvastatin $10 \mathrm{mg}$ daily, pravastatin $40 \mathrm{mg}$ daily, and simvastatin $20 \mathrm{mg}$ or $40 \mathrm{mg}$ daily. A total of 32,752 patients were followed up for a mean of 4.9 years. End points included the rate of new-onset diabetes and adverse CV events (defined as CV death, nonfatal MI, nonfatal stroke, coronary artery bypass graft surgery, and percutaneous coronary intervention). The rate of incident diabetes was statistically significantly higher with intensive statin therapy (OR 1.12, 95\% CI 1.04$1.22, I^{2}=0 \%$ ), which equates to two additional cases of diabetes per 1,000 patients per year (NNH of 498 per year) compared with moderate statin therapy. The risk of developing diabetes with intensive-dose simvastatin was similar to that of intensivedose atorvastatin (OR 1.13 and 1.15, respectively). The rate of adverse CV events was significantly lower with intensive statin therapy (OR $0.84,95 \%$ CI $0.75-0.94, I^{2}=74 \%$ ), which equals 6.5 fewer CV events per 1,000 patients per year with a NNT of 155. Overall, if 1,000 patients were treated for 1 year with intensive-dose statin therapy, compared with moderate-dose therapy, there would be approximately seven fewer CV events but two new cases of incident diabetes.

In 2012, a post hoc subgroup analysis of the Women's Health Initiative (WHI) was published investigating statin use and diabetes in women. ${ }^{6}$ The WHI enrolled 161,808 postmenopausal women between the ages of 50 and 79 years, 
who were followed up for approximately 6.5 years. Five percent of women were excluded from the analysis due to a history of diabetes at baseline, because of missing data, or because they were taking cerivastatin (withdrawn from the international market in 2001 due to reports of rhabdomyolysis). Only $7 \%$ of the 153,840 women were on statin therapy at baseline. Taking a statin at baseline was associated with a statistically significantly higher incidence of diabetes with a multivariate-adjusted HR of 1.48 (95\% CI 1.38-1.59). The association was consistently observed with all types of statins. When compared with non-use, both lowand high-potency statins were associated with an increased risk of diabetes (HR 1.48 and 1.45, respectively).

On February 28, 2012, the FDA released a Drug Safety Communication announcing label changes to all statin drugs. ${ }^{7}$ The label changes included information that "reports of increased blood sugar and glycosylated hemoglobin (HbAlc) levels [have] been added to the statin labels." Furthermore, the FDA stated that they "believe that the CV benefits of statins outweigh these small increased risks." In January 2013, a parallel announcement was made by Health Canada.

In order to validate this debate, it is crucial for one to consider the evidence regarding the benefits of statin therapy. Multiple randomized controlled trials have consistently demonstrated that statins reduce $\mathrm{CV}$ events despite this increased risk of diabetes. This is true for both patients with or without established cardiovascular disease (CVD). Trials investigating intensive-dose versus moderate-dose statin therapy in patients with CAD have demonstrated a reduction in major CV events by an absolute risk reduction (ARR) of approximately $2-4 \%$ $(\mathrm{NNT}=25-50) .{ }^{8,9} \mathrm{~A}$ meta-analysis by the Cholesterol Treatment Trialists' (CTT) Collaboration demonstrated that statin therapy in patients with or without CVD $(N=129,526)$, compared with placebo, reduced the risk of any major vascular event (defined as coronary death, nonfatal MI, coronary revascularization, and stroke) by $0.8 \%$ over 4.8 years (rate ratio [RR] $0.78,95 \%$ CI $0.76-$ 0.81). ${ }^{10}$ Additionally, more intensive versus less intensive statin therapy $(N=39,612)$ was also associated with an ARR of $0.8 \%$ in any major vascular event (RR $0.85,95 \%$ CI 0.82-0.89). Another meta-analysis investigating statins in patients with no history of CVD observed an ARR in major coronary events (defined as death from coronary heart disease and nonfatal MI) of $1.3 \%$ (OR $0.70,95 \%$ CI $0.61-0.81$ ) over 4.1 years, which equals an NNT of 77. ${ }^{11}$ Additionally, statins reduced death from any cause by an ARR of $0.6 \%$ (OR $0.88,95 \%$ CI $0.81-0.96$ ) with an NNT of 167. It is questionable as to whether the increased risk of diabetes with statin therapy increases CV risk, as atorvastatin has been demonstrated to reduce CV events in patients with established type 2 diabetes (ARR 3.2\% over 3.9 years, NNT =
32). ${ }^{12}$ To provide perspective, this CV benefit is far greater than that of any antihyperglycemic therapy.

\section{Benefit-Risk Assessment}

Based on the best available evidence, the truth appears to be that statins are associated with an increased risk of diabetes, and a higher dose appears to correspond with a higher risk. This relationship is likely a real effect as the trials were conducted in randomized populations. Therefore, both the intervention and comparator groups had an equal likelihood of developing diabetes based on other factors such as diet and inactivity. This is further substantiated by the low statistical heterogeneity observed in the meta-analyses presented. In addition, the likelihood of reporting bias by physicians is low as all of the studies were conducted in a double-blind fashion with appropriate allocation concealment. However, the evidence is not sufficient to conclude a causal relationship. Despite this, statins reduce $\mathrm{CV}$ events in multiple patient populations, including those with or without CVD and those with type 2 diabetes. Therefore, the most relevant issue to consider is whether this increased risk of diabetes should alter the use of statin therapy in practice. The quick answer to this is "no," but it should depend on the patient's baseline risk of CVD. In patients with established CAD, intensive-dose statin therapy over 4 years, compared with moderate-dose therapy, is associated with a CV event $\mathrm{NNT}$ of about $40,{ }^{5,8}$ whereas the $\mathrm{NNH}$ for diabetes is about $125^{5}$ (Table 1). Therefore, for every 1,000 patients with CAD treated with intensive-dose statin therapy, about 25 major CV events would be prevented but eight patients would develop diabetes. It is not known what the long-term sequelae are for patients who develop diabetes secondary to statin therapy, specifically the microvascular complications. Overall, it appears that the increased risk of macrovascular complications with diabetes (i.e., $\mathrm{MI}$, stroke) is mitigated by statin therapy. The benefit-versus-risk assessment for statin therapy in primary prevention is similar. The NNT to prevent a major coronary event with statin therapy in patients without CVD over 4 years is roughly $77,{ }^{11}$ compared with the NNH for diabetes of $255 .{ }^{4}$ Therefore, 1,000 primary prevention patients treated with statin therapy, compared with placebo, would result in roughly 13 fewer coronary events and four new cases of diabetes. Of note, the risk-versus-benefit assessment of statin therapy does not extend beyond the length of the published trials, which on average is about 4 years.

\section{Conclusion}

Statins reduce CV events and all-cause mortality in patients with or at risk for CVD. However, emerging data also suggest that statins are associated with new-onset diabetes. We want the truth: 
Table 1. Comparison of NNT and NNH with Statin Therapy

\begin{tabular}{lll} 
& $\begin{array}{l}\text { NNT to Prevent 1 CV/Coronary } \\
\text { Event over 4 Years }\end{array}$ & $\begin{array}{l}\text { NNH to Cause 1 Case of } \\
\text { Incident DM over } 4 \text { Years }\end{array}$ \\
\hline $\begin{array}{l}\text { Intensive-dose vs. moderate-dose statin } \\
\text { therapy in patients with CAD }\end{array}$ & 40 & 125 \\
\hline $\begin{array}{l}\text { Statin therapy vs. placebo in patients with } \\
\text { no history of CVD }\end{array}$ & 77 & 255 \\
\hline
\end{tabular}

$\mathrm{CAD}=$ coronary artery disease; $\mathrm{CV}=$ cardiovascular; $\mathrm{CVD}=$ cardiovascular disease; $\mathrm{DM}=$ diabetes mellitus; $\mathrm{NNH}=$ number needed to harm; NNT = number needed to treat.

the risk of diabetes with statins appears to be real, and this should be incorporated into the therapeutic decision-making process. This is of particular relevance for younger patients who are at low risk for CVD. Can we handle this truth? This debate can be simplified by comparing the potential benefits of statin therapy with the potential risks, while incorporating clinical judgment and the patient's own values and preferences into the final decision. As recommended by the FDA, patients should be made aware that statin therapy may result in a small but realistic increase in the risk of diabetes. However, this should not change current practice, as the beneficial CV effects of statin therapy appear to exceed the risk of developing diabetes.

\section{References}

1. The Associated Press. Statins linked with small diabetes risk. Toronto: Canadian Broadcasting Corporation, 2012; http://www.cbc.ca/news/health/story/2012/01/10/statins-diabetes.html. Accessed March 15, 2012.

2. Ridker PM, Danielson E, Fonseca FAH, et al. Rosuvastatin to prevent vascular events in men and women with elevated C-reactive protein. N Engl J Med 2008;359:2195-207.

3. Waters DD, Ho JE, DeMicco DA, et al. Predictors of new-onset diabetes in patients treated with atorvastatin. J Am Coll Cardiol 2011;57:1535-45.

4. Sattar N, Preiss D, Murray HM, et al. Statins and risk of incident diabetes: a collaborative meta-analysis of randomised statin trials. Lancet
2010;375:735-42.

5. Preiss D, Seshasai SRK, Welsh P, et al. Risk of incident diabetes with intensive-dose compared with moderate-dose statin therapy. JAMA 2011;305(24):2556-64.

6. Culver AL, Ockene IS, Balasubramanian R, et al. Statin use and risk of diabetes mellitus in postmenopausal women in the Women's Health Initiative. Arch Intern Med 2012;172(2):144-52.

7. US Food and Drug Administration. FDA drug safety communication: important safety label changes to cholesterol-lowering statin drugs. Silver Spring (MD): The Administration, 2012;

http://www.fda.gov/Drugs/DrugSafety/ucm293101.htm. Accessed March $16,2012$.

8. LaRosa JC, Grundy SM, Waters DD, et al. Intensive lipid lowering with atorvastatin in patients with stable coronary disease. $\mathrm{N}$ Engl J Med 2005;352:1425-35.

9. Cannon $\mathrm{CP}$, Braunwald $\mathrm{E}, \mathrm{McCabe} \mathrm{CH}$, et al. Intensive versus moderate lipid lowering with statins after acute coronary syndromes. N Engl J Med 2004;350:1495-504.

10. Cholesterol Treatment Trialists' (CTT) Collaboration. Efficacy and safety of more intensive lowering of LDL cholesterol: a meta-analysis of data from 170000 participants in 26 randomised trials. Lancet 2010;376:1670-81.

11. Brugts JJ, Yetgin T, Hoeks SE, et al. The benefits of statins in people without established cardiovascular disease but with cardiovascular risk factors: meta-analysis of randomised controlled trials. BMJ 2009;338:b2376.

12. Colhoun HM, Betteridge DJ, Durrington PN, et al. Primary prevention of cardiovascular disease with atorvastatin in type 2 diabetes in the collaborative atorvastatin diabetes study (CARDS): multicentre randomised placebo-controlled trial. Lancet 2004;364:685-96. 